\title{
The scaling of the density of states in systems with resonance states
}

\author{
Federico M Pont, Omar Osenda, and Pablo Serra \\ Facultad de Matemática, Astronomía y Física, Universidad Nacional de Córdoba, \\ and IFEG-CONICET, Ciudad Universitaria, X5016LAE Córdoba, Argentina \\ E-mail: serra@famaf.unc.edu.ar \\ E-mail: osenda@famaf.unc.edu.ar \\ E-mail: serra@famaf.unc.edu.ar
}

\begin{abstract}
Resonance states of a two-electron quantum dot are studied using a variational expansion with both real basis-set functions and complex scaling methods. We present numerical evidence about the critical behavior of the density of states in the region where there are resonances. The critical behavior is signaled by a strong dependence of some features of the density of states with the basis-set size used to calculate it. The resonance energy and lifetime are obtained using the scaling properties of the density of states

PACS numbers: 31.15.ac,03.67.Mn,73.22.-f
\end{abstract}




\section{Introduction}

Resonance states are slowly decaying scattering states characterized by a large but finite lifetime [1, 2]. There is a host of established methods that allow the calculation of the energy and lifetime associated to the resonance [1, 2, 3, 4, 5, 6, 7] . In many cases of interest where complex scaling (analytic dilatation) techniques can be applied, resonances energies and lifetimes show up as real and imaginary part, respectively, of isolated complex eigenvalues of the rotated Hamiltonian [1. Though conceptually simple, complex scaling is difficult to implement in molecular systems, for this kind of systems the analytical continuation is obtained using absorbing potential methods [8], which can be roughly grouped in two categories: the absorbing potentials based on negative imaginary potentials (NIPs), and the potentials derived from the complex scaling based theories (see [8] and References therein).

Despite the wealth of methods available to deal with the calculation of the resonance energy the subject constantly receives attention and new methods are proposed. The new proposals arise, mainly, because almost all the methods have some drawbacks or because new concepts are applied to the problem.

Recently Pont et al [9] and Ferrón et al [10] have used the Fidelity [11] and the von Neumann entropy [12], respectively, to obtain the real part of the resonance energy of a two electron quantum dot. Both concepts, borrowed from the quantum information theory, provided accurate methods for the calculation of resonance-state properties. In particular the method proposed by Pont et al [9] have some points in common with the work of Kar and Ho [7], both methods rest on the availability of a monoparametric family of basis sets, where each basis set corresponds to a particular value of a free parameter.

Kar and Ho [7] calculate a variational approximation for the problem which provides an approximate spectrum. With the spectrum they construct the density of states (DOS) of the problem and, after this step, it is possible to find the complex eigenenergy of the resonance fitting the DOS. Their method is quite general but the fit that is necessary to obtain the resonance energy is by no means unambiguously defined. In contradistinction the methods proposed by Pont et al [9] do not require any fitting, but to produce the resonance energy for a given problem (with its corresponding parameters) it is necessary to find an adequate value of the basis-set free parameter by bisection.

In this work we will present a method to find the energy of a resonance state using

the DOS but without resort to any fitting or bisection. We will study the resonance state that arise in a two electron quantum dot when its ground state looses stability and enters into the continuum. In Section 2 we briefly present the model and the variational method used to find an approximate spectrum. In Section 3 the DOS is obtained and the energy and lifetime of the resonance state are founded using finite size scaling techniques. Finally, in Section 4 we discuss our results and draw some conclusions. 


\section{The model and basic results}

There are many models of quantum dots, with different symmetries and interactions (see [4, 10] and References therein). In this work we consider a model with spherical symmetry, with two electrons interacting via the Coulomb repulsion. The main results should not be affected by the particular potential choice as it is already known that the near threshold behavior and other critical quantities (such as the critical exponents of the energy and other observables) are mostly determined by the range of the involved potentials [13]. Therefore to model the dot potential we use a short-range potential suitable to apply the complex scaling method. After this considerations we propose the following Hamiltonian $H$ for the system

$$
H=-\frac{\hbar^{2}}{2 m} \nabla_{\mathbf{r}_{1}}^{2}-\frac{\hbar^{2}}{2 m} \nabla_{\mathbf{r}_{2}}^{2}+V\left(r_{1}\right)+V\left(r_{2}\right)+\frac{e^{2}}{\left|\mathbf{r}_{2}-\mathbf{r}_{1}\right|},
$$

where $V(r)=-\left(V_{0} / r_{0}^{2}\right) \exp \left(-r / r_{0}\right), \mathbf{r}_{i}$ the position operator of electron $i=1,2 ; r_{0}$ and $V_{0}$ determine the range and depth of the dot potential. After re-scaling with $r_{0}$, in atomic units, the Hamiltonian of Eq. (1) can be written as

$$
H=-\frac{1}{2} \nabla_{\mathbf{r}_{1}}^{2}-\frac{1}{2} \nabla_{\mathbf{r}_{2}}^{2}-V_{0} e^{-r_{1}}-V_{0} e^{-r_{2}}+\frac{\lambda}{\left|\mathbf{r}_{2}-\mathbf{r}_{1}\right|},
$$

where $\lambda=r_{0}$.

We choose the exponential binding potential to take advantage of its analytical properties. In particular this potential is well behaved and the energy of the resonance states can be calculated using complex scaling methods. So, besides its simplicity, the exponential potential allows us to obtain independently the energy of the resonance state and a check for our results. The threshold energy, $\varepsilon$, of Hamiltonian Eq. (2), that is, the one-body ground state energy can be calculated exactly [14] and is given by

$$
J_{2 \sqrt{2 \varepsilon}}\left(\sqrt{2 V_{0}}\right)=0
$$

where $J_{\nu}(x)$ is the Bessel function.

The discrete spectrum and the resonance states of the model given by Eq. (2) can

be obtained approximately using $\mathcal{L}^{2}$ variational functions [4], [6]. So, if $\left|\psi_{j}(1,2)\right\rangle$ are the exact eigenfunctions of the Hamiltonian, we look for variational approximations

$$
\left|\psi_{j}(1,2)\right\rangle \simeq\left|\Psi_{j}(1,2)\right\rangle=\sum_{i=1}^{M} c_{i}^{(j)}\left|\Phi_{i}\right\rangle, \quad c_{i}^{(j)}=\left(\mathbf{c}^{(j)}\right)_{i} ; j=1, \cdots, M .(4)
$$

where the $\left|\Phi_{i}\right\rangle$ must be chosen adequately and $M$ is the basis set size.

The functions $\left|\Phi_{i}\right\rangle$ are chosen as eigenfunctions of the total angular momentum with zero eigenvalue. The radial part of the basis functions are constructed by symmetrization of the one-body Slater functions

$$
\phi_{n}^{(\alpha)}(r)=\left[\frac{\alpha^{2 n+3}}{(2 n+2) !}\right]^{1 / 2} r^{n} e^{-\alpha r / 2}
$$


With these constraints the functions $\left|\Phi_{i}\right\rangle$ have only three relevant quantum numbers $n_{1}, n_{2}$ and $l$, where $n_{1}$ and $n_{2}$ are related to the radial part of the function, and $l$ to the angular one [9]. The basis set is characterized by a non-linear parameter $\alpha$, then we use the notation $\left|\Phi_{i}\right\rangle \rightarrow\left|n_{1}, n_{2} ; l ; \alpha\right\rangle$. So, we get that

$$
\left|\Psi_{j}^{(\alpha)}(1,2)\right\rangle=\sum_{n_{1} n_{2} l} c_{n_{1} n_{2} l}^{(i),(\alpha)}\left|n_{1}, n_{2} ; l ; \alpha\right\rangle
$$

where $n_{1} \geq n_{2} \geq l \geq 0$, then the basis set size is given by

$$
M=\sum_{n_{1}=0}^{N} \sum_{n_{2}=0}^{n_{1}} \sum_{l=0}^{n_{2}} 1=\frac{1}{6}(N+1)(N+2)(N+3),
$$

so we refer to the basis set size using both $N$ and $M$. The matrix elements of the kinetic energy, the Coulombic repulsion between the electrons and other mathematical details involving the functions $\left|n_{1}, n_{2} ; l ; \alpha\right\rangle$ are given in references [9, [15], [16].
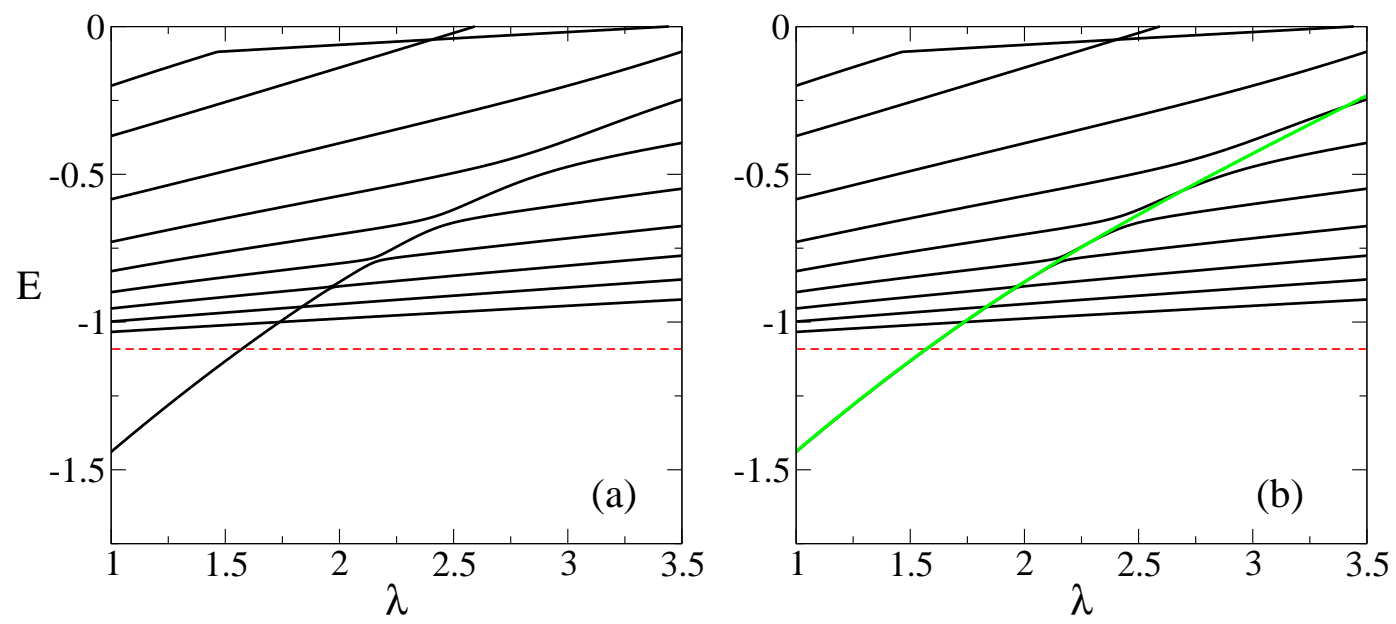

Figure 1. (color on-line) (a) the figure shows the behavior of the variational eigenvalues $E_{j}^{(\alpha)}(\lambda)$ (black lines) for $N=14$ and non-linear parameter $\alpha=2$. The red dashed line corresponds to the threshold energy $\varepsilon \simeq-1.091$. Note that the avoided crossings between the variational eigenvalues are fairly visible. (b) The figure shows the same variational eigenvalues that (a) (black lines) and the energy calculated using the complex scaling method (green line) for a parameter $\theta=\pi / 10$.

Resonance states have isolated complex eigenvalues, $E_{r e s}=E_{r}-i \Gamma / 2, \quad \Gamma>0$, whose eigenfunctions are not square-integrable. These states are considered as quasibound states of energy $E_{r}$ and inverse life time $\Gamma$. For the Hamiltonian Eq. (2), the resonance energies belong to the interval $(\varepsilon, 0)$ [2].

The resonance states can be analyzed using the spectrum obtained with a basis of $\mathcal{L}^{2}$ functions (see [10] and References therein). The levels above the threshold have several avoided crossings that "surround" the real part of the energy of the resonance state. The presence of a resonance can be made evident looking at the eigenvalues obtained numerically. Figure 1 shows a typical spectrum obtained from the variational method. This figure shows the behavior of the variational eigenvalues $E_{j}^{(\alpha)}$ as functions 
of the parameter $\lambda$, the results were obtained using $N=14$ and $\alpha=2.0$. The value of $\alpha$ was chosen in order to obtain the best approximation for the energy of the ground state in the region of $\lambda$ where it exists. The figure shows clearly that for $\lambda<\lambda_{t h} \simeq 1.54$ there is only one bound state. Above the threshold there is not a clear cut criteria to choose the value of the non-linear parameter, and the variational approximation provides a finite number of solutions with energy below zero. However, it is possible to calculate $E_{r}(\lambda)$ calculating $E_{j}^{(\alpha)}$ for many different values of the variational parameter (see Kar and Ho [7]).

Figure 2 shows the numerical results for the second eigenvalue $E_{2}^{(\alpha)}(\lambda)$, for different values of the variational parameter $\alpha$. As can be seen from Figure 2, there are three regions, in each one of them the curve for a given value of $\alpha$ is basically a straight line and the slope is different in each region. A feature that appears rather clearly is that, for fixed $\lambda$, the density of levels per unit energy is not uniform, despite that the curves $E_{j}^{\left(\alpha_{i}\right)}(\lambda)$ are drawn for forty equally spaced $\alpha_{i}$ 's between $\alpha=2.0$ and $\alpha=6.0$. This fact has been observed previously [17] and the DOS can be written in terms of two contributions, a localized one and an extended one. The localized DOS is attributed to the presence of the resonance state, conversely the extended DOS is attributed to the continuum of states between $(\varepsilon, 0)$.

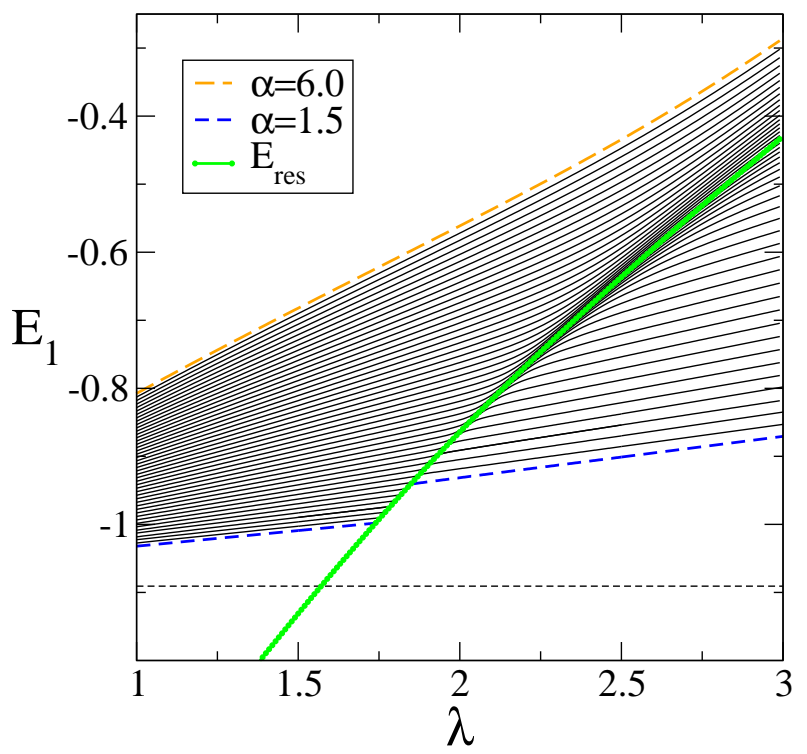

Figure 2. (color on-line) The second variational state energy vs $\lambda$, for different values of the variational parameter $\alpha$. From bottom to top $\alpha$ increases its value from $\alpha=2$ (dashed blue line) to $\alpha=6$ (dashed orange line). The real part of the resonance eigenvalue obtained using complex scaling $(\theta=\pi / 10)$ is also shown (green line). 


\section{The density of states}

The localized DOS $\rho(E)$ can be expressed as [7, 17]

$$
\rho(E)=\left|\frac{\partial E(\alpha)}{\partial \alpha}\right|^{-1} .
$$

Since we are dealing with a variational approximation, we calculate

$$
\rho\left(E_{j}^{\left(\alpha_{i}\right)}(\lambda)\right)=\left|\frac{E_{j}^{\left(\alpha_{i+1}\right)}(\lambda)-E_{j}^{\left(\alpha_{i-1}\right)}(\lambda)}{\alpha_{i+1}-\alpha_{i-1}}\right|^{-1} .
$$

In complex scaling methods the Hamiltonian is dilated by a complex factor $\eta=\tilde{\alpha} e^{-i \theta}$. As was pointed out long time ago by Moiseyev and coworkers, the parameter $\tilde{\alpha}$ is equivalent to our parameter $\alpha$ [18]. Besides, the DOS attains its maximum at optimal values of $\alpha$ and $E_{r}$ that could be obtained with a self-adjoint Hamiltonian without using complex scaling methods [19]. So, locating the position of the resonance using the maximum of the DOS is equivalent to the stabilization criterium used in complex dilation methods that requires the approximate fulfillment of the complex virial theorem [20].

Figure 3 shows the typical behavior of $\rho_{j}(E) \equiv \rho\left(E_{j}^{\left(\alpha_{i}\right)}(\lambda)\right)$ for several eigenenergies, with $\lambda=2.25$. The real and imaginary parts of the resonance's energy, $E_{r}(\lambda)$ and $E_{i}(\lambda)=-\Gamma / 2$ respectively, can be obtained from $\rho(E)$, see for example [7] and references therein.

The values of $E_{r}(\lambda)$ and $\Gamma(\lambda)$ are obtained performing a nonlinear fitting of $\rho(E)$, with a Lorentzian function,

$$
\rho(E)=\rho_{0}+\frac{A}{\pi} \frac{\Gamma / 2}{\left[\left(E-E_{r}\right)^{2}+(\Gamma / 2)^{2}\right]} .
$$

One of the drawbacks of this method results evident: for each $\lambda$ there are several $\rho_{j}(E)$ (in fact one for each variational level, see Figure 3 ), and since each $\rho_{j}(E)$ provides a value for $E_{r}^{j}(\lambda)$ and $\Gamma^{j}(\lambda)$ one has to choose which one is the best. Kar and Ho [7] solve this problem fitting all the $\rho_{j}(E)$ and keeping as the best values for $E_{r}(\lambda)$ and $\Gamma(\lambda)$ the fitting parameters with the smaller $\chi^{2}$ value. At least for their data the best fitting (the smaller $\chi^{2}$ ) usually corresponds to the larger $n$. This fact has a clear interpretation, if the numerical method approximates $E_{r}(\lambda)$ with $E_{n}^{(\alpha)}(\lambda)$ a large $n$ means that the numerical method is able to provide a large number of approximate levels, and so the continuum of states between $(\varepsilon, 0)$ is "better" approximated.

In most numerical studies it is assumed that using the largest possible basis set size leads to the best results. Indeed, most usually this assumption is correct but taking in consideration only the results obtained for the largest basis set size does not provide any insight about the accuracy of the result.

The Finite Size Scaling method (FSS) provides a systematic way to analyze the data generated using different basis set sizes and has been extensively used in atomic physics [21], statistical mechanics [22], etc. The FSS method requires a scaling function 


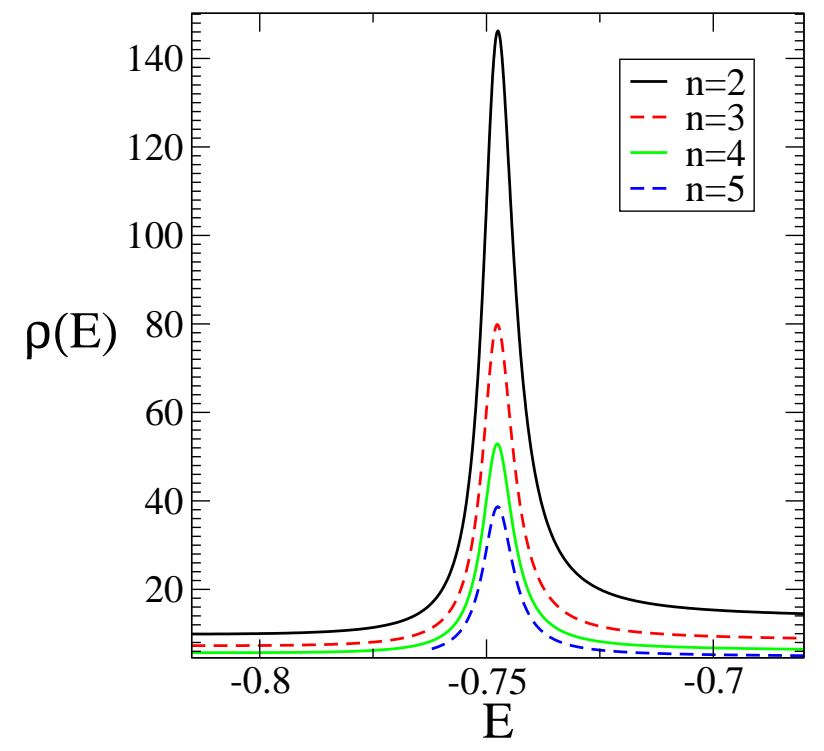

Figure 3. (color on-line) The DOS $\rho(E)$ for $\lambda=2.25$ and basis set size $N=14$. The results were obtained using Eq. (8) and correspond, from top to bottom, to the second (black line), third (dashed red line), fourth (green line) and fifth (dashed blue line) levels.

that reduces the data corresponding to different basis set sizes to a single "universal" set. Moreover, as we will show, the FSS allows to obtain the real and imaginary parts of the resonance energy using any $\rho_{j}(E)$.

We chose $\rho_{3}(E)$ to apply the FSS method, but using any other $\rho_{j}$ should lead to the same conclusions. As we have said previously the functional form of the scaling function is unique, independently of the actual value of $\lambda$. Figure 4(a) shows $\rho_{3}(E)$ for different values of $\lambda$ and several basis set sizes. For a fixed value of $N$, say $N=16$ the Figure 4(a) shows seven peaks, each one corresponding to a given value of $\lambda$. The values of $\lambda$ were chosen to sample different regimes of the resonance, from small values -sharp resonances- to larger ones -broad resonances. The broadening of the peaks corresponds roughly to the broadening of the resonance or, equivalently, to larger values of $\Gamma$.

Figure 4(b) shows the scaled data of panel (a), for each $\lambda$ the curves corresponding to different values of $N$ collapse into a single one which is independent of $N$.

The form of the scaling function can be inferred from the behavior of the maximum value of the function $\rho_{3}(E)$ for different basis set sizes. Figure 5 shows the behavior of $\ln \left(\rho_{3 \max }\right)$ vs $\ln (N)$, for $N$ large enough the maximum values behave as a power law. With this numerical evidence we propose

$$
\rho(E) \longmapsto N^{-\beta(\lambda)} \rho(E)
$$

as the scaling function for the DOS. As can be seen in Figure 4 (b), the collapse of the data is excellent. The exponent $\beta$ depends on $\lambda$ and its value must be found for each case. So, for a given $\lambda$ the real part of the complex resonance energy corresponds to the value where the DOS attains its maximum, $E_{r}(\lambda)=E_{\rho_{\max }}$, where 

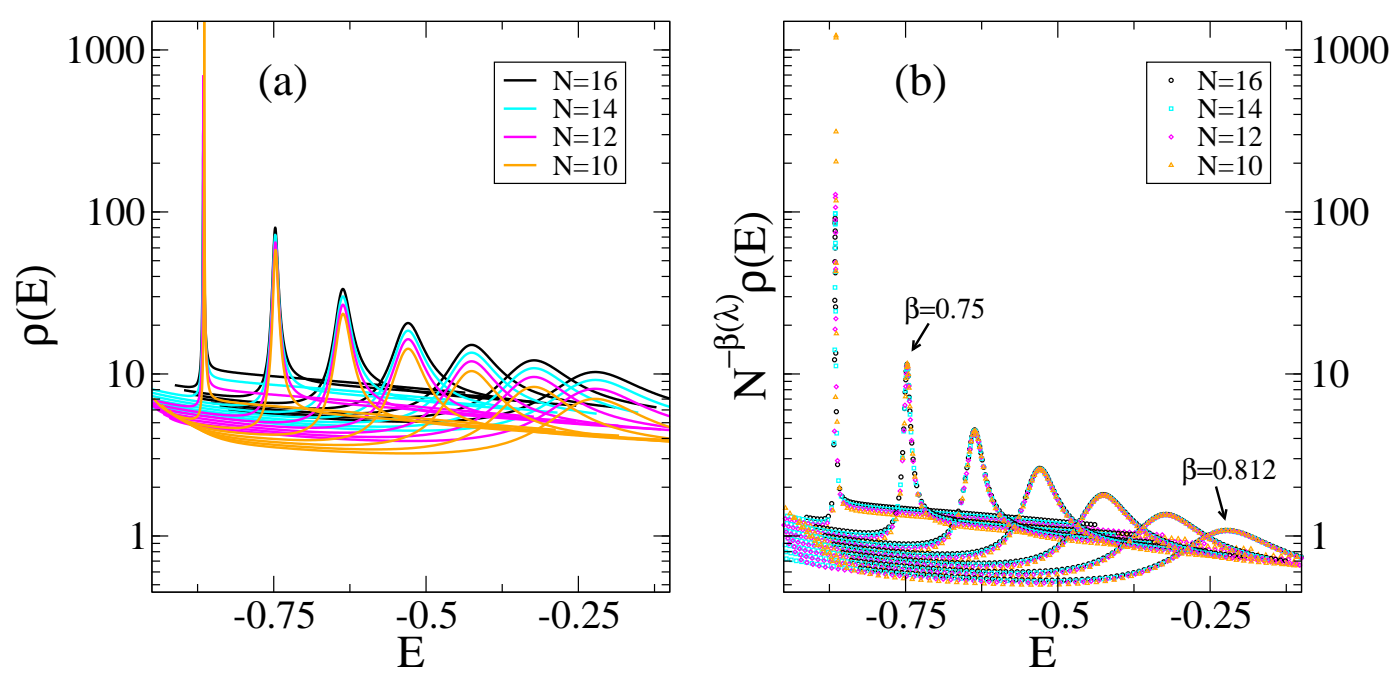

Figure 4. (color on-line)(a) The $\operatorname{DOS} \rho_{3}(E)$ for (from left to right) $\lambda=$ $2.25,2.50,2.75,3.00,3.25$ and 3.50 and different basis set sizes. The results were obtained using Eq. (8). (b) Data collapse obtained using the scaling function $N^{-\beta(\lambda)} \rho(E)$

$\rho_{\max }=\max _{E} \rho(E)=\rho\left(E_{\rho_{\max }}\right)$. At least from our data it is clear that the localization of the maximum of the DOS is a very slowly function of the basis set size, see Figure 5 (b).
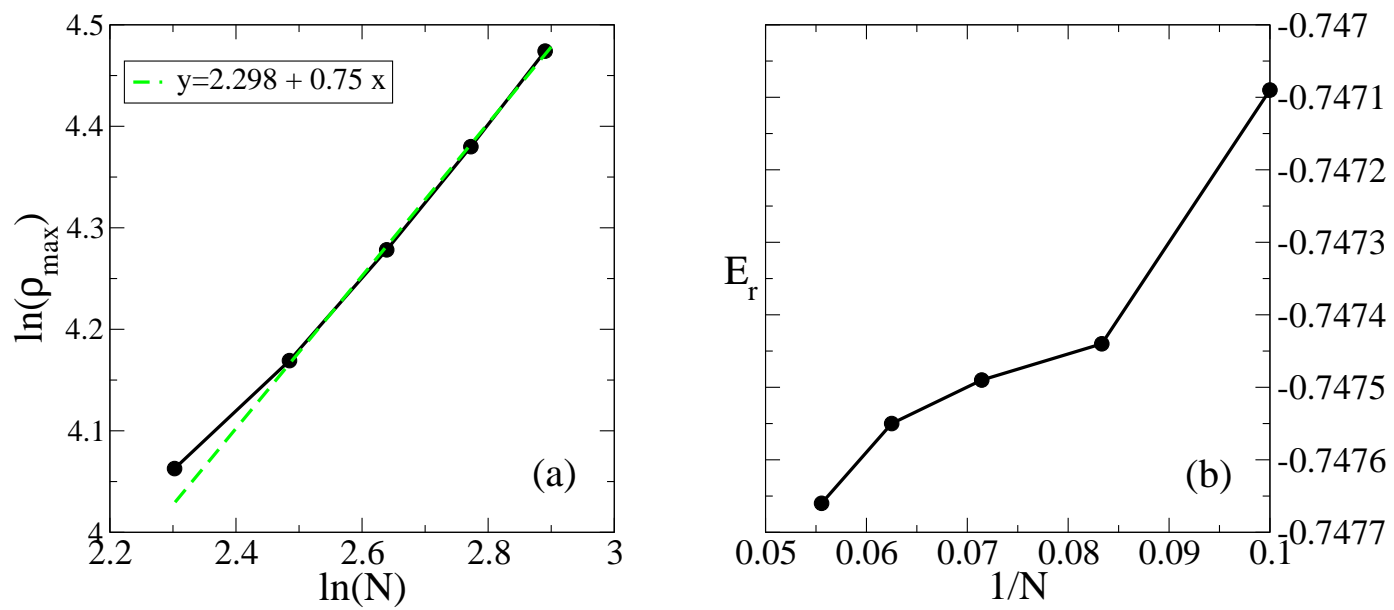

Figure 5. (color on-line) (a) $\log$ - $\log$ plot of the maximum of the density of states against the basis-set size $N$. (b) $E_{r}$ vs. $1 / N$. For both panels the data were obtained with $\lambda=2.25$.

On the following we will argue about the form of the scaling function necessary to obtain the imaginary part of the resonance energy, $\Gamma / 2$. As we have said previously, the real part of the resonance energy is located between $(\varepsilon, 0)$, and as $\log$ as $\Gamma \rightarrow 0$ when $\lambda \rightarrow \lambda_{c}^{+}$, the scaling function proposed for the imaginary part of the resonance energy should be zero at $\lambda_{c}$ or, equivalently, $E_{r}\left(\lambda_{c}\right)=\varepsilon$, and $E_{i}\left(\lambda_{c}\right)=-\Gamma\left(\lambda_{c}\right) / 2=0$. Besides, 
in some examples the life time of a resonance is proportional to the DOS, $\Gamma \propto \frac{1}{\rho}$, so we tested our numerical results using

$$
E_{i}^{(N)}(\lambda)=-\kappa\left(E_{r}^{(N)}(\lambda)-E_{c}\right) N^{\delta} \frac{1}{\rho_{\max }^{(N)}},
$$

where we have made explicit the superscript $N$ to emphasize the dependency with the basis set size, kappa is a positive constant, and $\delta$ is a exponent to be determined. As a first guess we used $\delta=0.8$, since this figure is very close to the exponents founded studying the scaling of the maximum of the DOS. Then, we observed that

$$
\frac{E_{i}^{(N)}\left(\lambda^{\prime \prime}\right)}{E_{i}^{(N)}\left(\lambda^{\prime}\right)} \simeq \frac{E_{i}^{(C S)}\left(\lambda^{\prime \prime}\right)}{E_{i}^{(C S)}\left(\lambda^{\prime}\right)},
$$

i.e. the ratio between the imaginary parts of two resonance energies, corresponding to $\lambda^{\prime \prime}$ and $\lambda^{\prime}$ chosen arbitrarily, calculated using the Complex Scaling (CS) method and Eq. (12) is fairly the same. Regrettably this finding does not provide the value of the constant $\kappa$. Just to compare with the result of Complex Scaling methods 9, we calculated $\kappa$ imposing that $E_{i}^{(N)}(\lambda)=E_{i}^{(C S)}(\lambda)$ for one particular $\lambda$. Figure 6 shows the values obtained for $E_{i}^{(N)}$ obtained using the recipe described above and Eq. (12), the agreement between values corresponding to different basis set sizes is excellent, and the same can be said with respect to the values obtained from Complex Scaling, see Figure 6 (a). Despite that the value of $\kappa$ was obtained using a particular value of $N$ and $\lambda$ our numerical findings strongly support the functional form proposed in Eq. (12), in Figure 6 (b) we present a comparison between the $E_{i}^{(N)}(\lambda)$ 's and the Complex Scaling results, the agreement is better than $1 \%$ for all the values of $\lambda$ and $N$ analyzed.
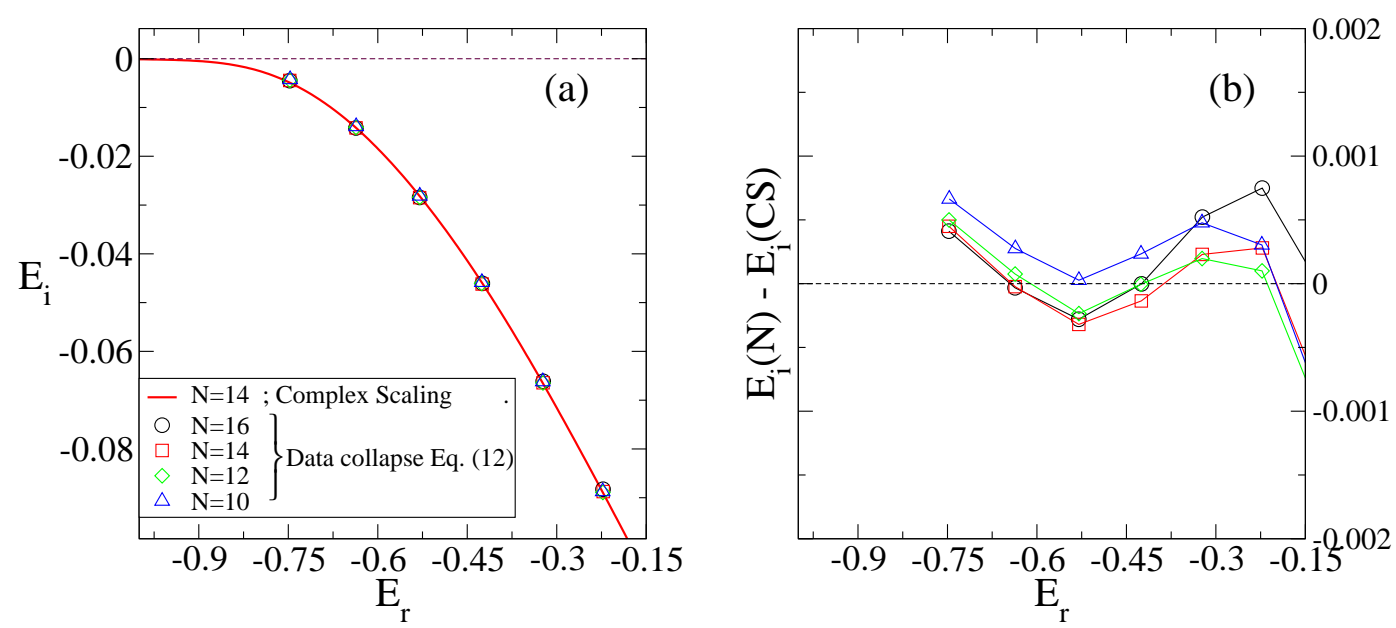

Figure 6. (color on-line) (a) Resonance pole string curve obtained from complex scaling and basis-set size scaling. A mean value of $\delta=0.8$ was chosen for the scaling exponent. (b) The energy discrepancies between the two methods. The relative error is less than $1 \%$ for the calculated values. 


\section{Summary and conclusions}

So far we have presented numerical evidence of the critical behavior of the density of states near the peak associated to the presence of a resonance state. The critical behavior should be understood in the sense of FSS method, i.e there is a strong dependency of the density of states with the basis set size used to obtain it. The critical behavior also manifest itself through the scaling properties of some quantities, in particular the maximum value attained by the density of states for fixed $\lambda$ and $N$.

Despite that we have been able to propose a scaling function that provides the imaginary part of the resonance energy, the proposal is made only in phenomenological terms and, more importantly, relays in other methods to be quantitative. Our efforts will be directed to put the scaling law Eq.(12) over a more formal basis, which hopefully will allow us to obtain the constants $\kappa$ and $\delta$ using only scaling arguments.

The critical behavior of the density of states studied in this work, together with

the behavior observed in the fidelity [9], points that the analogy between the "line" of resonance states and a "critical line" (in the sense of critical phenomena) could be more exploited and it is worth of further exploration.

\section{Acknowledgments}

We would like to acknowledge SECYT-UNC, CONICET and FONCyT for partial financial support of this project.

\section{References}

[1] N Moiseyev, Phys. Rep. 302, 211 (1998).

[2] W P Reinhardt and S Han, Int. J. Quantum Chem. 57, 327 (1996).

[3] Y Sajeev and N Moiseyev, Phys. Rev. B 78, 075316 (2008).

[4] M Bylicki, W Jaskólski, A Stachów and J Diaz, Phys. Rev. B 72, 075434 (2005).

[5] J Dubau and I A Ivanov, J. Phys. B: At. Mol. Opt. Phys. 313335 (1998).

[6] A T Kruppa and K Arai, Phys. Rev. A 59, 3556 (1999).

[7] S Kar and Y K Ho, J. Phys. B: At. Mol. Opt. Phys. 37, 3177 (2004).

[8] Y Sajeev, V Vysotskiy, L S Cederbaum, and N Moiseyev, J. Chem. Phys. 131, 211102 (2009)

[9] F M Pont, O Osenda, P Serra and J H Toloza, arXiv:1003.0468 [Phys. Rev. A (accepted for publication 2010)].

[10] A Ferrón, O Osenda and P Serra, Phys. Rev. A 79, 032509 (2009).

[11] P Zanardi and N Paunković, Phys. Rev. E 74, 031123 (2006).

[12] M A Nielsen and I L Chuang, Quantum Computation and Quantum Information, Cambridge University Press; first edition (September 2000).

[13] F M Pont and P Serra, J. Phys A: Math. Theor. 41, 275303 (2008).

[14] A Galindo and P Pascual. Quantum Mechanics I ( Springer, 1991).

[15] O Osenda, P Serra and S Kais, Int. J. Quantum Inf. 6, 303 (2008).

[16] P Serra and S Kais, Chem. Phys. Lett. 372, 205 (2003).

[17] V A Mandelshtam, T R Ravuri and H S Taylor, Phys. Rev. Lett. 70, 1932 (1993).

[18] N Moiseyev, C Corcoran, Phys. Rev. A 20, 814 (1979).

[19] N Moiseyev, S Friedland, Phys. Rev. A 22, 618 (1980). 
[20] N Moiseyev, S Friedland, P R Certain, J. Chem. Phys. 78, 4739 (1981)

[21] S Kais and P Serra, Adv. Chem. Phys. 125, 1 (2003), and references therein.

[22] M E Fisher, In Critical Phenomena, Proceedings of the 51st Enrico Fermi Summer School, Varenna, Italy, M. S. Green, ed. (Academic Press, New York 1971). 